\section{Allergy \\ Immunology}

Published online: September 5, 2013

\title{
Associate Editor Carsten B. Schmidt-Weber
}

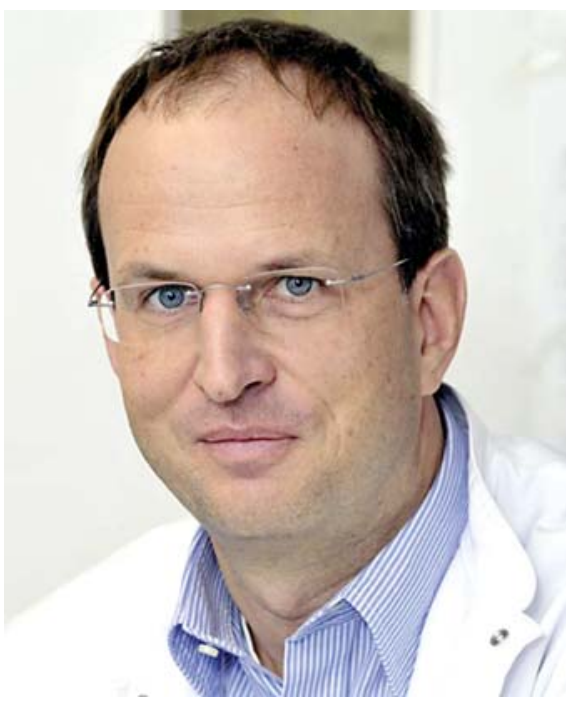

Carsten B. Schmidt-Weber, Munich.
Prof. Dr. rer. nat. Carsten B. Schmidt-Weber's main interests are the mechanisms of allergy, allergen tolerance and its clinical translation. His teams are also investigating environmental factors such as pollen, allergens and pollution with their impact on health and immune priming, mechanisms of skin and airway allergies as well as the development of new therapy approaches.

He qualified at the Technical University in Darmstadt and Friedrich Alexander University Erlangen-Nuremberg and conducted his biology diploma (1992) and PhD thesis (1996) at the Max Planck Research Unit for Immunology and Rheumatology in Erlangen. His postdoctoral work on IL-4 and IL-10 gene regulation and immune tolerance was performed at the Pathology Department, Brigham and Women's Hospital, Harvard Medical School. Prof. Schmidt-Weber was appointed by the Swiss Institute of Allergy and Asthma Research and founded the Department of Molecular Immunology (1998). His thesis on 'Molecular mechanisms of T-lymphocyte regulation by transforming growth factor beta' was awarded with the venia legendi in experimental immunology by the Medical Faculty of the University of Zurich, Switzerland (2006), and he joined the Imperial College in the year 2007. On April 1, 2010, Prof. Schmidt-Weber accepted the Chair of Molecular Allergology at the Medical Faculty of the Technical University of Munich. In 2011, he became Institute Director at the Helmholtz Center Munich to convert the Center for Allergy and Environment (ZAUM) into a Technical University of MunichHelmholtz hybrid to orient the center towards a translational research institute. The Helmholtz affiliations allow large-scale approaches and helped integrating the Center for Allergy and Environment into the national research efforts, specifically into the German Lung Center (DZL). Prof. Schmidt-Weber is managing the virtual network of the Munich Allergy Research Center (MARC; http://www.marc-allergy.de/), which is a large allergy research cluster covering more than 25 research groups in Munich. Prof. Schmidt-Weber is also involved in biotechnology startup/small enterprises that aim to trans- 
late basic research findings into clinical practice. The strategy is to form alliances with Universities and large research clusters such as the Helmholtz Center Munich to support pharmaceutical developments in the field of allergy, which is insufficiently addressed by large pharmaceutical companies.

Prof. Schmidt-Weber is a member of the Executive Committee of the $\mathrm{PhD}$ Graduate School for Biomolecular Medicine, Faculty of Medicine, Technical University of Munich, and gives lectures and practical courses for MD and $\mathrm{PhD}$ fellows in molecular immunology and medicine. Prof. Schmidt-Weber is a member of the prestigious Collegium Internationale Allergologicum as well as Chair of the Immunology section and member of the executive comitee of the EAACI. Furthermore, he supports the German Allergy Society (DGAKI) in the Extended Board and the Immunology Interest Group. 